\title{
The patient's medication access journey: a conceptual framework focused beyond adherence
}

\author{
Lee Holland, PharmD, MPH; Mel L Nelson, PharmD, CPHQ; Kimberly Westrich, MA; Patrick J Campbell, PharmD, PhD; \\ and Matthew K Pickering, PharmD
}

\section{What is already known about this subject}

- Medication access is a complex, multidimensional issue that must consider not only patient-specific challenges (eg, social determinants of health), but also health system limitations, among others.

- Several health care access and utilization frameworks have been created to illustrate the key relationships and interdependencies between elements of the system.

- None of the existing health accessibility frameworks focus exclusively on the unique journey that patients navigate while attempting to access medication.

\section{ABSTRACT}

BACKGROUND: It is well known that medication accessibility reduces morbidity and mortality and increases health-related quality of life; however, despite efforts to improve health care access, many Americans still face challenges in accessing medications. Several health care access and utilization conceptual frameworks have been created and used for decades to illustrate key relationships and interdependencies between elements of the system. However, none of these frameworks have focused exclusively on medication access and associated factors. Medication access is a complex, multidimensional issue that must consider not only patient-specific

\section{What this study adds}

- This study develops a framework that defines a patient's medication access journey and provides a patient-focused, holistic view of key nodes through which a patient must navigate in the health care system and beyond to access medication.

- The framework is cyclic in nature, beginning with a patient's awareness of an illness or condition, which induces a need to seek care (perceived need) and culminates with adherence to treatment (adherence) and includes reentry into the cycle if needed.

- This framework may be useful in payer beneficiary access analyses, public health and health services research, and quality measurement targeting improved medication access.

challenges, but also health system limitations, among others. A better understanding of medication access, beyond the proxy marker of adherence, is needed to identify opportunities to improve accessibility.

OBJECTIVE: To develop a conceptual framework that defines a patient's medication access journey and characterizes barriers frequently encountered while seeking medication access.

METHODS: A multistakeholder roundtable composed of 15 experts from across the health care continuum was convened in 2018 by the Pharmacy Quality Alliance to develop a conceptual framework for medication access. The roundtable participants were

\author{
Author affiliations \\ Lee Holland, PharmD, MPH; Mel L Nelson, \\ PharmD, CPHQ; Patrick J Campbell, PharmD, \\ PhD; and Matthew K Pickering, PharmD, \\ Pharmacy Quality Alliance, Alexandria, \\ VA. Kimberly Westrich, MA, National \\ Pharmaceutical Council, Washington, DC.
} AUTHOR CORRESPONDENCE: Mel L Nelson, mnelson@pqaalliance.org

J Manag Care Spec Pharm. 2021;27(12):1627-35

Copyright $\odot 2021$, Academy of Managed Care Pharmacy. All rights reserved.

convened through in-person and telephonic meetings. To inform their work, 2 literature reviews and an environmental scan were conducted to identify medication access barriers, interventions affecting medication access, and medication access quality measures.

RESULTS: The resulting framework included 7 nodes that represent the major access points encountered by patients when attempting to access medications: perceived need, help seeking, encounter, prescribing, prescription adjudication, prescription dispensing, and adherence. Also, 18 barriers were identified. Patient health literacy, cost, insurance, and organizational health literacy were predominant barriers across multiple nodes. 
CONCLUSIONS: The framework that was developed provides a patient-focused, holistic view of medication access, incorporating access nodes and corresponding barriers. It also provides a structure to consider key opportunities for interventions and measurement to address medication access challenges.

The US health care system is continuing to shift from volume-based to value-based care delivery with the goal of providing better, more affordable care to people and communities. One of the aims of the Patient Protection and Affordable Care Act (ACA), enacted in 2010, was to increase health care access for adults by improving the affordability of health services. ${ }^{1}$ Improving access to care is also a recognized priority of the National Quality Strategy. ${ }^{2}$ However, despite efforts to improve health care access, many Americans still face challenges accessing medications, ${ }^{3}$ even though it is well known that medication accessibility reduces morbidity and mortality and increases health-related quality of life. ${ }^{4-7}$ Accordingly, medication access continues to be of keen interest and concern to patients, providers, and policymakers. ${ }^{8}$

In this evolving, value-based health care landscape, quality performance measurement plays a critical role in the assessment and incentivization of the system. ${ }^{9,10}$ The current quality measure landscape consists of a variety of measures targeting medications, but few are focused on medication accessibility. ${ }^{11}$ Instead, they are predominantly developed and implemented in the area of adherence (eg, patient adherence to prescribed medications). ${ }^{11}$ Yet, adherence only captures a fraction of the medication use picture and fails to capture upstream financial (eg, cost of medication) and nonfinancial (eg, provider availability, health literacy) factors that may affect a patient's ability to access medication (ie, direct and indirect costs).

Medication access is a complex, multidimensional issue that must consider not only patient-specific challenges (eg, social determinants of health), but also health system limitations, among others. A better understanding of medication access, beyond the proxy marker of adherence, is needed to identify opportunities for quality performance measurement to incentivize the system toward improved accessibility. To accomplish these efforts, a consistent definition and framework characterizing medication access complexities is needed..$^{12-14}$

Several health care access and utilization conceptual frameworks have been created and used for decades to identify and target gaps in care. Three widely used frameworks include the Andersen Behavioral Model, the
Institute of Medicine Model of Access Monitoring, and the Derose Conceptual Framework for Public Health's Role in Addressing Disparities in Health Care Access. ${ }^{12-14}$ The Andersen Behavioral Model focuses on patient behavior in accessing and using health care, and although it has been adapted and expanded in various ways, its applications continue to focus on individual-level factors that contribute to access..$^{12}$ The Institute of Medicine model for monitoring access to personal health care services expands on the factors influencing health care access beyond individual-level characteristics and includes 11 barriers across structural, financial, and personal challenges. ${ }^{13}$ The Derose framework incorporates the role of the public health system in accessing health care, including public health agencies and programs overlaid with the community-level variables presented in previous frameworks. ${ }^{14}$

Yet, none of the health care access and utilization frameworks to date have focused exclusively on medication access and associated factors. Therefore, the objective of this study was to develop a conceptual framework to explicitly define medication access and associated barriers. A medication access framework has utility in identifying opportunities for quality measure development, research, and quality improvement that has the potential to be used to assess and incentivize appropriate medication access. Pharmacy Quality Alliance (PQA), a national quality organization dedicated to improving medication safety, adherence, and appropriate use, initiated the project to address, through a consensus-based process, the medication access framework gap. The study was led by PQA staff in partnership with other study team members.

\section{Methods}

This project was conducted in 2 phases: (1) information gathering and synthesis to inform framework development and (2) multistakeholder, collaborative roundtable to develop a conceptual framework specific to medication access.

\section{PHASE I: INFORMATION GATHERING AND SYNTHESIS}

An environmental scan and 2 literature reviews were conducted to provide background information for the roundtable participants convened in the second phase of the project. ${ }^{11,15}$

Literature Review 1: Medication Access Barriers. The purpose of this literature review was to provide information to the roundtable regarding common challenges and barriers patients face while attempting to gain access to medications in a post-ACA enactment health care system. A MEDLINE search was conducted to identify articles addressing 
medication access barriers that were published in English between January 1, 2010, and September 24, 2017 (date of search). The search was constructed using major Medical Subject Headings (MeSH) and key search terms associated with the following concepts: (1) health care accessibility and availability, (2) barriers and challenges to accessing health care, (3) medication availability, and (4) disparities in access to medications. Articles were excluded if they did not address the US health care system. Two reviewers independently screened titles and abstracts for topic relevance and then assessed full text for those that were not excluded during the initial relevance screen. A third reviewer resolved discrepancies.

The same process was followed for data extraction. Medication access barriers were extracted from articles qualified for inclusion, and barrier definitions were drawn from several sources, including 2 Institute of Medicine working groups, the National Healthcare Quality and Disparity Reports by the Agency for Healthcare Research and Quality, the Andersen Behavioral Model of Health Services Use, and the Derose public health framework..$^{12-17}$ The resulting list of medication access barriers and definitions was provided to the roundtable to inform framework development. ${ }^{15}$

Environmental Scan: Medication Access Quality Measure Landscape. An environmental scan was conducted to further understand the medication access barriers addressed in the current quality measurement landscape. ${ }^{11}$ Three quality measure databases were searched in January 2018: Centers for Medicare \& Medicaid Services (CMS) Measure Inventory Tool, Agency for Healthcare Research \& Quality National Quality Measures Clearinghouse, and National Quality Forum Quality Positioning System. Search terms included access, accessibility, accessible, availability, available, access to care, access to treatment, and access to medication. Two independent reviewers screened measures to determine if they were related to medication access directly (eg, needed medication received) or indirectly (eg, insurance coverage) and/or included a patient-perceived need to obtain care. A third reviewer resolved discrepancies.

The same process was used to map the medication access barriers identified in the first literature review to the measures selected for inclusion through the environmental scan. Measure rationale, title, and description were used to evaluate which medication access barriers could be addressed by each measure. In some cases, a measure was deemed to address multiple medication access barriers (eg, enrollment in a drug assistance program could address cost, medical condition, and insurance barriers). These results were provided to the roundtable to provide insight into medication access quality measurement gaps.

Literature Review 2: Interventions Impacting Medication Access. Quality measures may take years to develop and implement in public reporting programs. As such, it is not unexpected that an exhaustive list of measures targeting medication access would be found in existing quality measure databases. To gain an understanding of how medication access interventions were being measured in research and pilot studies, a MEDLINE (PubMed) search of peer-reviewed literature published in English between January 1, 2010, and October 2, 2018, was conducted. The search strategy was constructed using major MeSH headings and key search terms for the following concepts: (1) health care accessibility/availability, (2) assistance programs, and (3) drug/ pharmaceutical. Two independent reviewers screened titles and abstracts for topic relevance and assessed full text for those that were not excluded during the initial relevance screen. A third reviewer resolved discrepancies.

Data extraction was conducted in the same manner. Articles were included if they described interventions affecting medication access and assessed either direct or indirect medication access outcomes (eg, medication adherence, health care resource utilization) and excluded if they did not focus on the US health care system. Forward and backward reference searches were conducted on articles that met inclusion criteria for the study. To provide an understanding of medication access barriers addressed, data extraction included study type, population, intervention type (eg, legislative change, pilot program) setting, and key findings. In addition, reviewers evaluated which medication access barriers were addressed by each intervention and provided results to the roundtable..$^{15}$

\section{PHASE II: ROUNDTABLE CONCEPTUAL FRAMEWORK DEVELOPMENT}

The study team of 6 researchers from various backgrounds (ie, public health, pharmacy, health services research, outcomes research, and health care quality) convened a roundtable of 15 subject matter experts to create a conceptual framework characterizing a patient's medication access journey and associated barriers frequently encountered while seeking access. Purposive sampling was used to recruit experts from a priori identified key categories to obtain representation from across the health care continuum, including patient advocacy organizations, public and private payers, research institutions, health care quality organizations, professional associations, patient assistance programs, and a health care access framework developer. ${ }^{15}$ 


\begin{tabular}{|c|c|}
\hline Node & Description \\
\hline Perceived need & - Patient awareness of illness/condition that infers the need to seek care. \\
\hline Help seeking & $\begin{array}{l}\text { - Attempting to schedule an appointment with a medical provider. } \\
\text { - Contacting an insurance plan for lists of in-network providers. } \\
\text { - Checking insurance coverage for specific disease states and/or treatments. }\end{array}$ \\
\hline Encounter & $\begin{array}{l}\text { - Patient interacts with health care provider. } \\
\text { - Health concerns are presented for assessment and for possible treatment. }\end{array}$ \\
\hline Prescribing & - A medical provider selects an appropriate medication (if needed) after an assessment (encounter). \\
\hline Prescription adjudication & $\begin{array}{l}\text { - Process of paying or denying a submitted prescription insurance claim after comparing with the patient's benefit } \\
\text { or coverage requirements. } \\
\text { - Medication utilization tools (eg, prior authorizations and step therapy) may disrupt the timeliness of receiving } \\
\text { medication. }\end{array}$ \\
\hline Prescription dispensing & - Encompasses the point of contact between a patient and the pharmacy that provides the medication. \\
\hline Adherence & - Following a health care provider's recommendations to take medication as prescribed. \\
\hline
\end{tabular}

The roundtable was convened through 1 in-person and 2 webinar meetings over a period of 4 months in 2018 to draft, revise, and refine the framework through an iterative, consensus-building process. Consensus building began in the initial day-long, in-person session, where the study team presented phase I findings and facilitated interactive sessions to collaboratively white-board key access points (ie, nodes) unique to medication access. Through continued facilitated discussion, the roundtable mapped the previously identified medication access barriers across each node.

Following this meeting, the study team developed a Delphi survey derived from the output of the in-person meeting (ie, nodes and associated medication access barriers). All roundtable participants completed the survey to build consensus on the predominant access barriers affecting each node as having either severe, moderate, or minimal impact on medication access by ranking the barriers associated with each node. The top 3 barriers identified for each node were selected by at least $80 \%$ of respondents. Participants were also given a free-entry response option for each node ranking for discussion at the first follow-up webinar meeting.

During the follow-up meeting, medication access barrier rankings from the survey were shared. In addition, facilitated discussion was conducted, driven by the freetext survey comments for each node. Participants further refined medication access barrier names and definitions (eg, should cost subsume insurance, use of "medical conditions" vs "physical limitations") to refine the framework.
Before approving the final draft, the roundtable recommended that the study team present the framework to one of their internal, preexisting panels of patient and caregiver advisors. Panel members were asked what barriers and challenges they have encountered while accessing medications. Then, when presented with the framework, the panel members were asked when and where they experienced barriers. Their input was aligned with the draft framework, and the framework was finalized.

\section{Results}

During the roundtable examination of the phase I findings, several key topics were discussed to inform the framework. The environmental scan revealed that significant gaps exist in the medication access quality measure landscape. ${ }^{11}$ Current measures were indistinct, focusing on a broad delivery of care (eg, whether patients received needed care or received care quickly), rather than medication access specifically, which further emphasized the roundtable's imperative to define a medication access conceptual framework. Upon exploring the common themes presented in the 2 literature reviews, ${ }^{15}$ roundtable participants came to a consensus: (1) key access points (ie, nodes) on the journey to obtain medications should be defined, and (2) the most common barriers at each access point should be described.

The resulting conceptual framework, named the Medication Access Patient Journey (MAPJ), consists of 7 nodes that patients encounter when attempting to access medication: perceived need, help seeking, encounter, prescribing, prescription adjudication, prescription 


\section{FIGURE 1 Medication Access Patient Journey Framework: Nodes of Access}

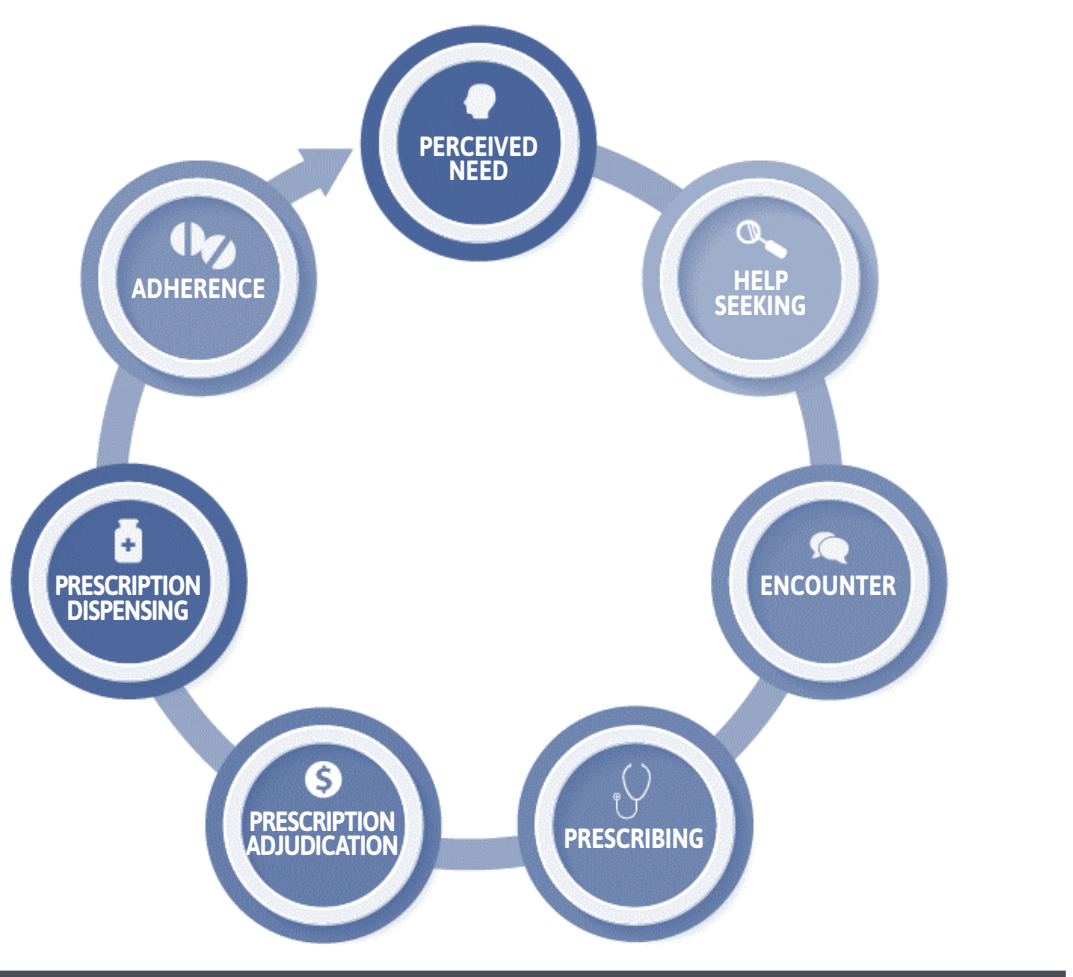

dispensing, and adherence. Each node is described in Table 1. As seen in Figure 1, the MAPJ is cyclic in nature, beginning with a patient's awareness of an illness or condition that induces a need to seek care (perceived need) and culminating with adherence to treatment (adherence). The framework also includes re-entry into the cycle or vascillation between nodes if needed. ${ }^{15}$

Roundtable participants formed consensus that the MAPJ should be designed as a cycle to account for complexities of long-term illnesses, misdiagnoses, and chronic diseases that might require patients to experience multiple iterations of the pathway. In addition, participants aimed to develop a holistic framework that includes health system-, provider-, and patient-level factors and examines the complex intersectionality of medication access barriers across each.

Many of the patient-level characteristics included in the framework center on social determinants of health. These factors, such as gender, race/ethnicity, income, and rural/urban place of residence, are mapped across all nodes of the MAPJ. Provider-level factors include specific competencies and beliefs of health care providers (eg, provider perception about appropriate treatment). Health system factors relate to transportation and local support for public health programs, as well as national and local health system structures.

Each of the identified medication access barriers across health system-, provider-, and patient-level factors were mapped in the MAPJ framework by the roundtable. As seen in Figure 2, patient health literacy is a predominant barrier, crossing all nodes apart from prescribing and dispensing. Direct (eg, out of pocket) and indirect (eg, time off work, day care, transportation) costs and insurance are significant barriers across the framework, touching all nodes except perceived need. Organizational health literacy is a major barrier in the prescribing, adjudication, and dispensing nodes, and other mapped barriers include transportation, insurance, and patient attitudes and beliefs. Each barrier is described in Table $2 .{ }^{15}$

\section{Discussion}

The MAPJ conceptual framework synthesizes and builds on existing health care access and utilization models in a comprehensive way to characterize medication access specifically. As with the Andersen model, the MAPJ incorporates sociobehavioral components across the framework and traces a patient's access journey by beginning with need and moving through the health ecosystem to outcome. Both models are cyclic in nature, which accounts for the influence of a patient's perceived health status after navigating the health ecosystem and how that perception may lead to ongoing future need and resultant system reentry. For the MAPJ specifically, this takes into consideration that patients may need to repeat several cycles of the framework due to long-term illnesses, misdiagnoses, medication adverse effects, lack of response to therapy, or chronic diseases.

In addition to focusing on patient behavior and characteristics, the MAPJ is holistic in that it includes an examination of the entire health ecosystem. This is similar to the Institute of Medicine and Derose models, given that both include 


\section{FIGURE 2 Most Common Access Barriers Mapped Across the MAPJ Framework}

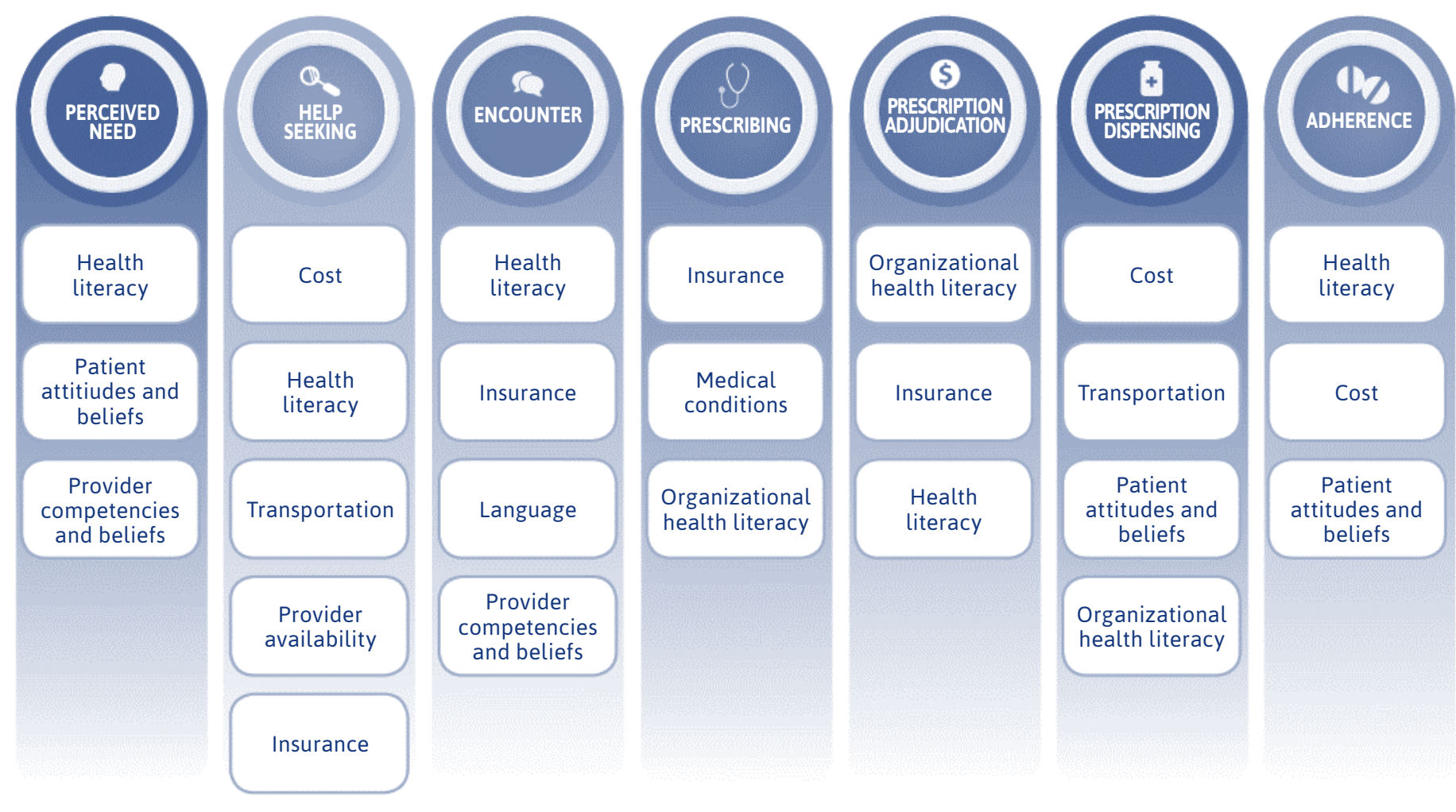

MAPJ= Medication Access Patient Journey

individual, financial, and structural barriers to accessing health care..$^{13,14}$ Consequently, the MAPJ framework allows for consideration of how the patient, local environment, and health care system interact. Furthermore, the MAPJ is the only framework that incorporates unique components of accessing medication specifically, such as obtaining a prescription, claim adjudication, medication dispensing, and adherence, representing an important advancement in the field. As such, it is a new tool to operationalize a holistic set of factors, including those upstream from adherence, that may affect a patient's ability to access medications.

Increased accessibility to health care, including medications, was a major goal of the ACA. ${ }^{1}$ Since the enactment of the ACA, quality measure development has increased ${ }^{18,19}$; however, very few existing measures address the complexity of medication access distinctly. ${ }^{11}$ Instead, medication adherence is often used as a proxy indicator of access and is consequently a frequent target of quality measures used within value-based programs (eg, adherence measures for diabetes, hypertension, and cholesterol medications included in the $2020 \mathrm{CMS}$ stars ratings program). ${ }^{20}$

Although adherence plays a critical role in quality improvement and performance measurement, it only provides information about patients who have already accessed the health ecosystem at some point. It does not inform barriers that a patient may encounter while attempting to obtain medications nor does it address upstream medication access initiatives. Until now, there has not been a single resource to help operationalize relevant targets for intervention or measurement. ${ }^{21,22}$

Because the MAPJ framework incorporates the various factors that can influence medication adherence, it can be used to identify and address complex barriers to obtaining medication. In addition, the holistic nature of the MAPJ framework allows for identification of barriers that are common throughout the patient journey that can be difficult to articulate when focusing on just 1 aspect of the patient-, provider-, health system interaction. 


\section{TABLE 2 Medication Access Barriers and Definitions}

Barrier

Organizational health literacy

Provider competencies and beliefs

Medical conditions

Health literacy

Insurance

Patient attitudes and

beliefs

Race/ethnicity
Gender

Provider availability

Language

Public support

Transportation

Rural/urban

Costs

Disability status

Income

Education
Definition

Organizational health literacy is how health literate health care systems are in providing patient care, which encompasses everything from management, organizational systems and interoperability, and the health care workforce.

How provider competencies and beliefs impact patient access to care (eg, lack of current medical knowledge, ability to provide culturally competent care, and outlook on stigmatized conditions).

Diseases and/or conditions that can impact access to health care.

Health literacy characterizes the capability of the public to obtain and understand health information. It also includes the ability to make health decisions and to navigate the health care system in order to obtain medical services.

Patient access to medical care/medications based on the type of medical insurance.

Patient attitude and values towards the health care system and how negative attitudes can lead patients to not utilizing medical services, creating a barrier to accessing care.

Racial or ethnic background and how it impacts access.

How gender impacts patient access to medical care.

Includes adequate medical infrastructure, facilities, and competent workforce to provide health care and medication after a need is identified.

Examination of barriers experienced by patients whose primary language is not English in gaining access to needed medical care.

Examines if taxpayer-funded health care programs provide access to certain forms of medical care.

Availability of transportation to medical care. This includes car ownership and the adequacy of public transportation.

Examines barriers that are unique to urban and rural communities.

The costs of obtaining health care services. This includes the indirect costs of receiving care (eg, transportation and time off work).

The unique issues that patients with disabilities can face in accessing health care and medications.

Barriers related to the income of patients.

Barriers related to patients' educational level.
These intersecting barriers can be helpful in revealing how social determinants of health play a role for patients when accessing the health care system. Although a focus on intersecting barriers across the MAPJ provides ample opportunity to identify and address access issues, the nodes also provide an area of emphasis for innovation. For example, the incorporation of prescription claim adjudication, medication dispensing, and adherence nodes point to the importance of pharmacy services in the multidisciplinary care team.

Pharmacists are medication experts, who provide medication management services, including assistance with navigating health insurance plans, prescription cost minimization, and adherence counseling, all of which can improve the safety and effectiveness of medication treatment and, ultimately, the quality of care..$^{23-28}$ In addition, pharmacists are one of the most accessible health care providers, which means they have a unique opportunity to address the complexities of medication access and educate to improve health literacy. ${ }^{29}$ Finally, pharmacies within the community provide a unique opportunity to collaborate with health care partners, including public health departments and community centers to implement community programs that address social determinants of health.

Overall, the MAPJ framework provides a structure around which further exploration (eg, research, quality improvement, quality measurement) can be conducted to improve medication access. Looking forward, health care stakeholders can leverage the MAPJ to identify key focal points and common, major barriers that can serve as targets for such efforts.

\section{LIMITATIONS}

This study has some limitations to consider. Purposive sampling is prone to research bias. As such, the study team used a priori identified categories for selection. The views of roundtable participants may not be reflective of all opinions, which may have affected the development of the final framework. In addition, the literature reviews on which this work is based were limited to articles published after enactment 
of the ACA; as such, it should not be seen as definitive. Furthermore, the context for this work was initially to inform quality measurement, so there may have been inherent biases in development.

\section{Conclusions}

The MAPJ framework provides a patient-focused, holistic view of the medication access journey, including access nodes and corresponding barriers. The 7 nodes within the framework represent the various stages that patients encounter when attempting to obtain a medication. The framework is cyclic in design, conveying that barriers and challenges occurring upstream of adherence can have a significant effect on medication access.

The holistic nature of the framework positions it as a tool for numerous health care stakeholders. For example, it can be useful in public health and health services research, payer beneficiary access analyses, and quality measurement targeting improved medication access. Development of access measures has the potential to drive the health care system toward incentivizing activities that improve access vs the system today that focuses primarily on adherence (eg, CMS star ratings).

Further work should map existing quality measures across the framework, identify key areas for new measure development, and explore innovative solutions to access challenges.

\section{DISCLOSURES}

This study was conducted with grant support from the National Pharmaceutical Council, which served as a collaborator in the study. Westrich is employed by the National Pharmaceutical Council. Nelson is employed by the Pharmacy Quality Alliance, which was contracted to conduct this study. Pickering, Campbell, and Holland were employed by the Pharmacy Quality Alliance at the time of this study.

This research was presented as a professional poster at the American Public Health Association Annual Meeting in October 2019, Philadelphia, PA.

\section{ACKNOWLEDGMENTS}

The authors acknowledge all members of the roundtable and study team for their contributions to the development of the framework described in this study.

\section{REFERENCES}

1. Patient Protection and Affordable Care Act. HR 3590, 111th Cong (2009-2010). Accessed February 14, 2020. https:// www.congress.gov/bill/111th-congress/ house-bill/3590

2. Agency for Healthcare Research and Quality. About the National Quality Strategy. Last reviewed March 2017. Accessed December 4, 2019. https://www. ahrq.gov/workingforquality/about/index. $\underline{\text { html }}$

3. Kirzinger A, Lopes L, Wu B, Brodie M. KFF Health Tracking Poll-February 2019: Prescription Drugs. Kaiser Family Foundation. March 1, 2019. Accessed October 25, 2019. https://www.kff. org/health-costs/poll-finding/ kff-health-tracking-poll-february2019-prescription-drugs/

4. Shulman NB, Levinson RM, Dever A, Porter RS, Owen SL, Hall WD. Impact of cost problems on morbidity in a hypertensive population. Am J Prev Med. 1991;7(6):374-78.

5. Spiker EC, Giannamore MR, Nahata MC. Medication use patterns and health outcomes among patients using a subsidized prescription drug program. J Am Pharm Assoc (2003). 2005;45(6):714-19.
6. Lichtenberg FR. The impact of new drug launches on longevity: evidence from longitudinal, disease-level data from 52 countries, 1982-2001. Int J Health Care Finance Econ. 2005;5(1):47-73.

7. Cremieux PY, Meilleur MC, Ouellette P, Petit P, Zelder M, Potvin K. Public and private pharmaceutical spending as determinants of health outcomes in Canada. Health Econ. 2005;14(2):107-16.

8. Department of Health and Human Services. HHS blueprint to lower drug prices and reduce out of pocket costs. Fed Regist. 2018;83(95):22692-700. Accessed February 14, 2020. https://www.govinfo.gov/content/pkg/FR-2018-05-16/ pdf/2018-10435.pdf

9. Fonarow GC, Abraham WT, Albert NM, et al. Influence of a performanceimprovement initiative on quality of care for patients hospitalized with heart failure. Arch Intern Med. 2007;167(14):1493-502.

10. Bardach NS, Wang JJ, De Leon SF, et al. Effect of pay-for-performance incentives on quality of care in small practices with electronic health records: a randomized trial. JAMA. 2013;310(10):1051-59.

11. Mehas N, Nelson M, Pickering M, Ashemore A, Westrich KD. PHP76 How are we measuring access to healthcare in the US? - an environmental scan of quality measures [abstract]. Value Health. 2018;21(1):S99. Accessed September 30, 2021. https://www.valueinhealthjournal. com/article/S1098-3015(18)30968-9/pdf

12. Andersen RM, Davidson PL. Improving access to care in America: individual and contextual indicators. In: Andersen RM, Rice TH, Kominski EF, eds. Changing the U.S. Health Care System: Key Issues in Health Services, Policy, and Management. Jossey-Bass; 2001:3-30.

13. Institute of Medicine. Access to Health Care in America. National Academies Press; 1993.

14. Derose KP, Gresenz CR, Ringel JS. Understanding disparities in healthcare access - and reducing them-through a focus on public health. Health Aff (Millwood). 2011;30(10):1844-51. doi: 10.1377/hlthaff.2011.0644 
15. Pharmacy Quality Alliance. Access to care: development of a medication access framework for quality measurement. White paper. March 2019. Accessed February 14, 2021. https://www. pqaalliance.org/assets/Research/PQAAccess-to-Care-Report.pdf

16. Brach C, Keller D, Hernandez LM, et al. Ten attributes of health literate health care organizations. National Academy of Sciences. June 2012. Accessed September 30, 2021. https://nam.edu/wp-content/ uploads/2015/06/BPH_Ten_HLit_ Attributes.pdf

17. Agency for Healthcare Research and Quality. National Healthcare Quality and Disparities Reports. Data query. Accessed October 30, 2018. https://nhqrnet.ahrq. gov/inhqrdr/data/query

18. Centers for Medicare \& Medicaid Services. Pre-Rulemaking. MAP reports. Accessed February 14, 2020. https://www.cms.gov/Medicare/ Quality-Initiatives-Patient-AssessmentInstruments/QualityMeasures/ Pre-Rulemaking\#MAP_Reports

19. Casalino LP, Gans D, Weber R, et al. US physician practices spend more than \$15.4 billion annually to report quality measures. Health Aff (Millwood). 2016;35(3):401-06.
20. Centers for Medicare \& Medicaid Services. Medicare 2020 Part C \& D star ratings technical notes. Updated October 1, 2019. Accessed February 14, 2020. https://www.cms.gov/ Medicare/Prescription-Drug-Coverage/ PrescriptionDrugCovGenIn/Downloads/ Star-Ratings-Technical-NotesOct-10-2019.pdf

21. Hafner T, Walkowiak H, AboagyeNyame F, et al. Defining pharmaceutical systems strengthening: concepts to enable measurement. Health Policy Plan. 2017;32(4):572-84.

22. Gotham D, Onarheim KH, Barber MJ. How the MDGs gave up on measuring access to medicines. Lancet Glob Health. 2016;4(5):e296-97.

23. Ko JJ, Lu J, Rascati K, et al. Analysis of glycemic control of a pharmacist-led medication management program in patients with type 2 diabetes. J Manag Care Spec Pharm. 2016;22(1):32-37. doi: 10.18553/jmcp.2016.22.1.32

24. Niznik JD, He H, Kane-Gill SL. Impact of clinical pharmacist services delivered via telemedicine in the outpatient or ambulatory care setting: a systematic review. Res Social Adm Pharm. 2018;14(8):707-17.
25. Horstmann E, Trapskin K, Wegner MV. The Wisconsin Pharmacy Quality Collaborative - a team-based approach to optimizing medication therapy outcomes. WMJ. 2014;113(3):95-98.

26. Ingram SJ, Kirkdale CL, Williams S, et al. Moving anticoagulation initiation and monitoring services into the community: evaluation of the Brighton and Hove Community Pharmacy Service. BMC Health Serv Res. 2018;18(1):91.

27. Doucette ED, Salas J, Scherrer JF. Insurance coverage and diabetes quality indicators among patients in NHANES. Am J Manag Care. 2016;22(7):484-90.

28. Westrick SC, Hastings TJ, McFarland SJ, Hohmann LA, Hohmann NS. How do pharmacists assist Medicare beneficiaries with limited income? A cross-sectional study of community pharmacies in Alabama. J Manag Care Spec Pharm. 2016;22(9):1039-45. doi: 10.18553/ jmcp.2016.22.9.1039

29. Tsuyuki RT, Beahm NP, Al Hamarneh YN, et al. Pharmacists as accessible primary health care providers: Review of the evidence. Can Pharm J (Ott). 2018;151(1):4-5. 